\title{
An improved multipath MANET routing using link estimation and swarm intelligence

\author{
Prabha R. ${ }^{*}$ and Ramaraj N. ${ }^{2}$
}

\begin{abstract}
Routing in ad hoc networks is challenging as nodes are mobile and links are continuously created and broken. Current on-demand ad hoc routing algorithms start route discovery after path break, incurring high cost to detect the disconnection and to establish a new route. Specifically, when a path is liable to break, the source is warned about the likelihood of disconnection. The source then starts path discovery avoiding disconnection entirely. A path is likely to break when link availability decreases. Since routing is nondeterministic polynomial (NP) hard, this work proposes an improved ad hoc on-demand multipath distance vector (AOMDV) based on link availability, neighboring node's queuing delay, node mobility, and bit error rate. The optimal path is selected using BAT meta-heuristic optimization. Simulation shows improved performance compared to AOMDV.
\end{abstract}

Keywords: Routing; Route discovery; Link availability; Ad hoc on-demand multipath distance vector (AOMDV); BAT optimization

\section{Introduction}

With emerging mobile applications [1-3], mobile ad hoc networks (MANETs) have attracted research from various groups due to its flexibility and usability in diverse applications. A MANET is a self-configuring temporary network of mobile nodes which are independent with each other and do not have any fixed infrastructure. MANETs do not control or regulate traffic [4] within the network but utilize the intermediate node's routing capability. Since source and destination nodes use intermediate nodes as routers, a routing path must be established for actual communication. Routing protocols are the key to MANET success and are an active area for MANET research [5-9].

Many routing protocols have been proposed for ad hoc networks in literature which find a route based on given criteria for packet delivery from source to destination. In literature, routing protocols are broadly classified as table-driven protocols and on-demand protocols. In the former, also called proactive routing protocols, every node maintains a table of data containing routing information such that source can reach any node in the

\footnotetext{
* Correspondence: prabha.aomdv@gmail.com

${ }^{1}$ Department of Electronics and Communication Engineering, Indira Gandhi College of Engineering and Technology for Women, Tamil Nadu, India Full list of author information is available at the end of the article
}

destination if a route exists. Popular table-driven protocols include optimized link state routing (OLSR) and destination sequenced distance vector (DSDV). In ondemand routing protocols, routes are created as and when needed. They are also called as reactive protocols, and the source invokes route discovery process when data has to be transmitted. A route is valid till destination is reached or until route is not required. Popular existing on-demand routing protocols include dynamic source routing (DSR) and ad hoc on-demand distance vector AODV $[10,11]$ protocol.

In AODV, [12, 13] a source node broadcasts route request (RREQ) to its neighbors. When adjacent nodes received RREQ with source node and target node addresses, it judges if it is the target. If yes, it sends a route reply (RREP); otherwise, it checks if it has active route to the destination in its table. If it has a fresh route, then it sends RREP to the source or it continues flooding by sending RREQ. AODV protocol discovers neighborhood nodes through regular broadcast of hello messages. When a link breaks, it sends route error message while deleted/broken records are repaired.

Ad hoc on-demand multipath distance vector (AOMDV) [14] is an AODV extension for computing multiple loopfree and link-disjoint paths. The routing table for destination includes a list of next hops and the number of hops 
to reach the destination. In AOMDV, all the available nexthop neighbors are assigned the same sequence numbers. A node maintains advertised hop count for every destination, and this hop count sends destination route advertisements. Every duplicate route advertisement that has been broadcasted and received by a node defines an alternative destination path.

A node accepts an alternative destination path-to ensure loop freedom-only when the hop count it has is lower than advertised hop count. To locate disjoint routes, a node does not reject duplicate RREQs immediately. Every RREQ has an additional field called first hop indicating its first hop. Also, a node maintains a firsthop list for every RREQ to track list of neighbors of source through which a RREQ copy was received. To ensure link disjoints in RREP's first hop, destination replies only to RREQs arriving through unique neighbors. Every RREP trajectory may intersect at an intermediate node, but each goes on a different reverse path to source ensuring link disjointness [15].

\subsection{Related works}

A multipath routing protocol proposed by Obaidat et al. [16] is a variant of single-path AODV routing protocol. The proposed method established node-disjoint paths with lowest delays based on interaction of factors from various layers. The proposed protocol's performance was investigated and compared to single-path AODV and multipath AOMDV protocols using Operations Network (OPNET). Results show improved performance of the proposed method in terms of throughput and end-toend delay.

Adaptive multi-metric (AM)-AOMDV, an improved AOMDV based on multiple metrics, was proposed by Khimsara et al., [17]. AM-AOMDV extends AOMDV by including many route metrics, a new local route update, and route maintenance algorithm. The latter uses onehop information exchange between one-hop nodes to improve route longevity. The modified technique improved packet delivery ratio and decreased end-to-end delay. Significant improvement was also found in route discovery frequency and routing overhead during node mobility. Multiple metrics ensure routing scheme convergence to most efficient route during transmission and avoided creation of hotspots.

Mallapur and Patil [18] proposed a Stable Backbonebased Multipath Routing Protocol (SBMRP). In the proposed protocol, candidate nodes were selected based on available bandwidth, link quality, node mobility, and available power. Source-destination paths were established through candidate nodes, to form a routing backbone. When a candidate node fails in a path due to lack measure values, another candidate node is used to create an alternate path. Simulation proved that the new technique reduced overhead, packet drop, and energy while increasing packet delivery rate.

Yu et al. [19] proposed Link Effective Available Time (LEAT). The method aimed to locate a link available time during epoch through measurement of distances between a mobile link's two nodes rather than using localization information. A new routing link cost was proposed which lowered link breakage. Routing was formulated as an optimal routing problem based on new cost, using a heuristic algorithm. Simulation shows that LEAT improved network performance in delay, hop counts, and throughput when compared to present routing algorithms.

Yu and Bao [20] proposed a new link cost to reduce breakages during data service. To reduce implementation complexity, the authors used only ranging information in link availability and avoided localization information. Routing was formulated, based on new metrics, as an optimal routing problem, needing a heuristic algorithm. Simulation proved that RBLAR improved link connectivity by reducing link breaks and improved network performance. Considering link reliability in route choice was proposed by Yelemou [21]. Route request procedure was modified to enable reliable paths using bit error rate (BER). Simulations showed the proposed system outperformed AOMDV even in conditions like mobility and multi-communication.

Chen et al., [22] extended AOMDV routing protocol to accommodate channel fading. The new channelaware AOMDV (CA-AOMDV) used channel average nonfading duration as routing metric to choose stable links for path discovery applying a pre-emptive handoff strategy to ensure reliable connection. Using this information, paths when available were reused and not discarded. The proposed system ensured lower downtime. The authors also showed via simulations common network performance measures, with good insights into differences in performance between CA-AOMDV and AOMDV.

Link availability estimation was used to select most stable route from alternate paths was proposed by Mazumdar et al. [23] and was implemented in AOMDV for route selection. Results show that selecting stable route leads to a higher throughput in dynamic network topologies.

Node mobility is a major factor link stability in MANETs. Cai and Liu [24] suggested Prediction of Link Stability-AOMDV (PLS-AOMDV) based on AOMDV multipath routing protocol that periodically predicted link stability by considering both node mobility and energy consumption to choose a high stability link. Simulation shows that PLS-AOMDV improved packet delivery rate.

Energy-efficient (EE)-AODV routing protocol that is an enhancement of current AODV routing protocol was 
proposed by Singh and Gupta [25]. Routing algorithm adopted by EE-AODV enhanced RREQ and RREP process to save mobile devices energy. EE-AODV considers a threshold energy level as minimum energy which must be available with a node for use as an intermediary node. When node energy reaches the threshold level, it would not be considered an intermediary node unless alternative paths are unavailable. Simulation shows that network life increased in EE-AODV compared to AODV.

Gunes et al. [26] proposed a swarm intelligence-based multipath routing protocol using ant colony optimization. The proposed algorithm reduced the routing overheads compared to traditional multipath routing protocol. Liu and Feng [27] used ant colony optimization metaheuristic on the node-disjoint multipath routing problem. The proposed algorithm concurrently sends data via multiple paths. Ziane and Melouk [28] extensively investigated multipath routing for multimedia data using swarm intelligence. Meta-heuristic techniques have been extensively used in wired and wireless networks including wireless sensor network, multicast routing [29-31].

Yen et al. [32] proposed a multi-constrained quality of service (QoS) multicast routing method using genetic algorithm. The proposal will be flooding-limited using the available resources and minimum computation time in a dynamic environment. By selecting the appropriate values for parameters such as crossover, mutation, and population size, the genetic algorithm improves and tries to optimize the routes. Simulation results indicate its better performances compared to other methods.

Song et al. [33] exploited a biological model of Physarum to design a novel biology-inspired optimization algorithm for minimal exposure problem (MEP). First, formulate MEP and the related models and then convert MEP into the Steiner problem by discretizing the monitoring field to a large-scale weighted grid. Inspired by the path-finding capability of Physarum, the authors developed a biological optimization solution to find the road-network with minimal exposure among multiple points of interest. POA can also be used for solving the general Steiner problem. Extensive simulations demonstrate that the proposed models and algorithm are effective for finding the road-network with minimal exposure and feasible for the Steiner problem [34].

$\mathrm{Li}$ et al. [35] proposed a reliable multicast protocol, called CodePipe, with advanced performance in terms of energy efficiency, throughput, and fairness in lossy wireless networks. Built upon opportunistic routing and random linear network coding, CodePipe not only simplifies transmission coordination between nodes but also improves the multicast throughput significantly by exploiting both intra-batch and inter-batch coding opportunities. In particular, four key techniques, namely LP-based opportunistic routing structure, opportunistic feeding, fast batch moving, and inter-batch coding, are proposed to offer substantial improvement in throughput, energy efficiency, and fairness. CodePipe was evaluated on NS2 simulator by comparing with other two state-of-the-art multicast protocols, MORE and Pacifier. Simulation results show that CodePipe significantly outperforms both of them.

The importance of routing in disruptive tolerant network, cognitive network, mesh network, and wireless sensor network has been extensively discussed in [33, 36-48]. From literature survey, it can be observed that link quality plays a very important role for obtaining better QOS in multipath routing protocols. Routing being nondeterministic polynomial (NP) hard, various meta-heuristic-based routing algorithms have also been proposed in literature using swarm intelligence metaheuristic. Popular meta-heuristic algorithms including genetic algorithm and particle swarm optimization (PSO) have shown to have drawbacks when the solution to be found is multimodal. In this work, it is proposed to investigate an improved AOMDV algorithm based on link quality and BAT meta-heuristic which has been shown to perform well for multimodal optimization problems.

\subsection{Methodology}

This work uses BAT optimization to find optimal route for AOMDV based on link quality and neighboring node queuing delay. In this section, the technique used for predicting link availability and queuing delay is presented. Since the problem is multi objective, BAT meta-heuristic is used to find optimal routes. For link availability estimation, the basic assumptions used are similar to that used in literature [49-54]. The movement of a node from one location to another at a constant speed and direction is called epoch. The mobility of the nodes is comprised of multiple epochs which need not be same. The different epoch size used in this work is different from the same epoch length used in literature. Assuming each epoch is exponentially distributed with mean $\lambda^{-1}$ such that

$$
\begin{aligned}
E(x) & \stackrel{\Delta}{=} P\{\text { Epoch length } \leq \mathrm{x}\} \\
& =1-\mathrm{e}^{-\lambda x}
\end{aligned}
$$

The node mobility among different nodes is uncorrelated. Assuming all nodes have the same average epoch length, it is possible to predict the link availability between two nodes for duration $T_{p}$ using equation (2) 


$$
L\left(T_{p}\right) \triangleq P\left\{t_{0} \text { to } \mathrm{t}_{0}+T_{p}=\text { available } \mid \text { when available at } t_{0}\right\}
$$

In case the node velocity changes between two epochs and the distance between the nodes is less than the maximum transmission range of the node, the link availability can be computed using equation (3).

$$
L\left(T_{p}\right)=L_{1}\left(T_{p 1}\right)+L_{2}\left(T_{p 2}\right)
$$

Since movement among nodes are independent and based on the assumption of exponential distribution it can be shown that

$$
\begin{aligned}
L_{i}\left(T_{p}\right) & =\left[1-E\left(T_{p i}\right)\right]^{2} \\
& =\mathrm{e}^{-2 \lambda T_{p i}}
\end{aligned}
$$

It is possible to find the direction of node movement and whether two nodes are moving towards each other or away from each other using received signal strength indication (RSSI). The link quality degrades the square of the distance between the nodes, as the nodes move from each other. Alternatively, the change between a fixed epoch length can be computed by assuming a random variable $\omega$ which is a value smaller than $t_{0}+T_{p}$, and assuming both nodes are moving away from each other, the link quality can be given by

$$
L_{s}(\omega)=\frac{\omega+\left(T_{p}-\omega\right)}{T_{p}}
$$

The average $L_{s}(\omega)$ can be computed by equation (6)

$$
\bar{L}_{s}=\int_{0}^{T_{p}} L_{s}(\omega) f(\omega) d \omega
$$

Where $f(w) \geq 0$ is given by

$$
\begin{aligned}
f(\omega) & =\lim \frac{P\left\{\omega \leq W<T_{p}\right\}-P\left\{\omega+\Delta \omega \leq W<T_{p}\right\}}{\Delta \omega} \\
& =2 \lambda \mathrm{e}^{-2 \lambda \omega}
\end{aligned}
$$

Substitute $L_{s}(\omega)$ and $f(w)$ with equation (3), we obtain equation (7)

$$
=\frac{1}{2 \lambda T_{p}}+e^{-2 \lambda T_{p}}\left(p \lambda T_{p^{-}}-\frac{1}{2 \lambda T_{p}}\right)
$$

In a multihop network, the average delay to deliver a packet from source to destination is dependent on the

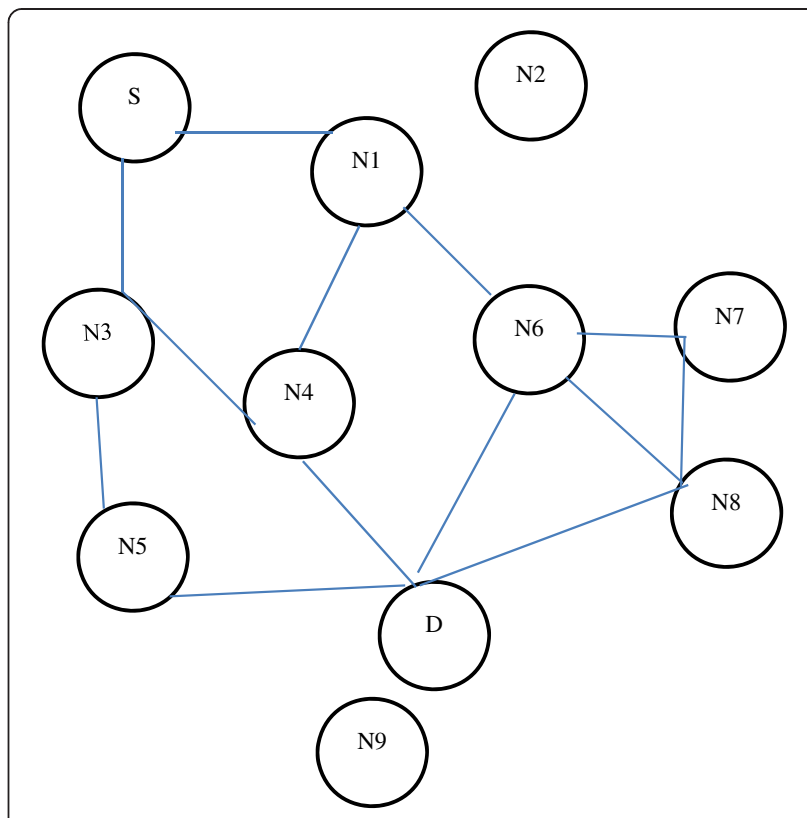

Fig. 1 A sample network with routes between source and destination

number of hops to the destination. Delay cumulatively increase based on the data processing time, queuing time, and transmission time. Propagation delay in an ideal medium can be assumed to be minimal as the transmission distance is not substantial in MANETs. Decreasing queuing delay load balances the network. For a given route, let $P_{i, j}(t)$ be the number of packets available between node $_{i}$ and nodej. For the time interval $[0,1]$, let the number of packets that arrive be given by $\alpha(t)$. If the time taken for the packet to leave node ${ }_{i}$ and reach node $_{j}$ is $T_{i}$, then the load in the link between $i$ and $j$ is given by equation (8)

$$
P_{T}=\frac{1}{T} \int_{0}^{t} P(\tau) d \tau
$$

$P_{T}$ changes with time and reaches a steady state as $t$ increases and can be given by equation (9)

$$
P=\lim _{t \rightarrow \infty} P_{T}
$$

Using Little's theorem, equation (9) can be solved and a probability-based model can also be derived. If routes

Table 1 BAT parameters used

\begin{tabular}{ll}
\hline BAT population & 10 \\
\hline Pulse frequency & {$[0.0,3.0]$} \\
Pulse emission & {$[0,1]$} \\
Loudness & 0.6 \\
Maximum number of generations & 500 \\
\hline
\end{tabular}


Table 2 Simulation parameters used in this work

\begin{tabular}{ll}
\hline MAC layer protocol & IEEE 802.11 \\
\hline Channel capacity & 2 Mbps \\
Number of nodes used in simulation & 60 \\
Transmission range of node & $250 \mathrm{~m}$ \\
Mobility & Random way point \\
Network size & $1200 \times 800 \mathrm{~m}$ \\
Traffic type & Constant bit rate (CBR) \\
Packet size & 512 bytes \\
\hline
\end{tabular}

are selected based only on link quality, load balancing becomes a major issue.

For a given source to destination, multiple routes are discovered using the link metric in AOMDV by modifying the route discovery process. To maintain a balance between the network load and QoS, the objective is to minimize

$$
f(x)=\frac{\operatorname{PLR}\left(P_{\mathrm{e}}\right)\left(\mathrm{e}^{-\left(\frac{d_{\mathrm{t}}}{d}\right)^{2}}\right)}{L_{\mathrm{e}}}
$$

where PLR is the packet loss rate, $L_{\mathrm{e}}$ is the estimated link, $P_{\mathrm{e}}$ is the estimated load, $d_{\mathrm{t}}$ is the delay threshold, and $d$ is the delay in route.

In this work, the BAT meta-heuristic is used to select the optimal route such that the objective is minimized. Xing-She Yang proposed BAT algorithm (BA) [55], which imitates micro bat's echolocation behavior. Bats are the only mammals that fly and have advanced echolocation capabilities using short-frequency modulated signals which help it to identify obstructions. Micro bat is an example though all species use echolocation extensively. Bats emit a loud sound pulse and listen to resulting echo which bounces back from surrounding objects. The algorithm is based on the way micro bats fly to find food and avoid objects. The process of BAT algorithm is given by:

1: Define objective function $f(x)$

2: Initialize the bat population $\left(\mathrm{x}_{\mathrm{i}}\right)$ and initial velocity $\left(\mathrm{v}_{\mathrm{i}}\right)$

$\mathrm{i}=(1 \ldots, n)$

3: Establish pulse frequency range $Q_{i} \in\left[Q_{\min }, Q_{\max }\right]$

4: Select initial pulse rates $r_{i}$ and the loudness $A_{i}$

5: while $\left(t<T_{\max }\right) / /$ number of iterations

6: $\quad$ Generate new solutions by adjusting frequency, and

7: $\quad$ updating velocities and locations/solutions

8: $\quad$ if $\left(\operatorname{rand}(0,1)>r_{i}\right)$

9: $\quad$ Select a solution among the best solutions

10: Generate a local solution around the best solution

11: end if

12: Generate a new solution by flying randomly

13: $\quad$ if $\left(\operatorname{rand}(0 ; 1)<\mathrm{A}_{\mathrm{i}}\right.$ and $\left.\mathrm{f}\left(\mathrm{x}_{\mathrm{i}}\right)<\mathrm{f}(\mathrm{x})\right)$

14: $\quad$ Accept the new solutions

15: Increase $r_{i}$ and reduce $A_{i}$

16: end if

17: Rank the bats and find the current best

18: end while

19: Post process results and visualization

From the pseudo code, it can be seen that initial $\mathrm{n}$ random solutions, which are feasible routes between source and destination, are defined. For the frequency and pulse rate, compute the objective for each solution. Find the best route and generate local solutions based on the best route. Fig. 1 shows an example of how a local solution is created.

$$
\begin{aligned}
& \text { Random solution created can be } \\
& \mathrm{S} \rightarrow \mathrm{N} 1 \rightarrow \mathrm{N} 6 \rightarrow \mathrm{N} 7 \rightarrow \mathrm{N} 8 \rightarrow \mathrm{D} \\
& \mathrm{S} \rightarrow \mathrm{N} 1 \rightarrow \mathrm{N} 4 \rightarrow \mathrm{D}
\end{aligned}
$$

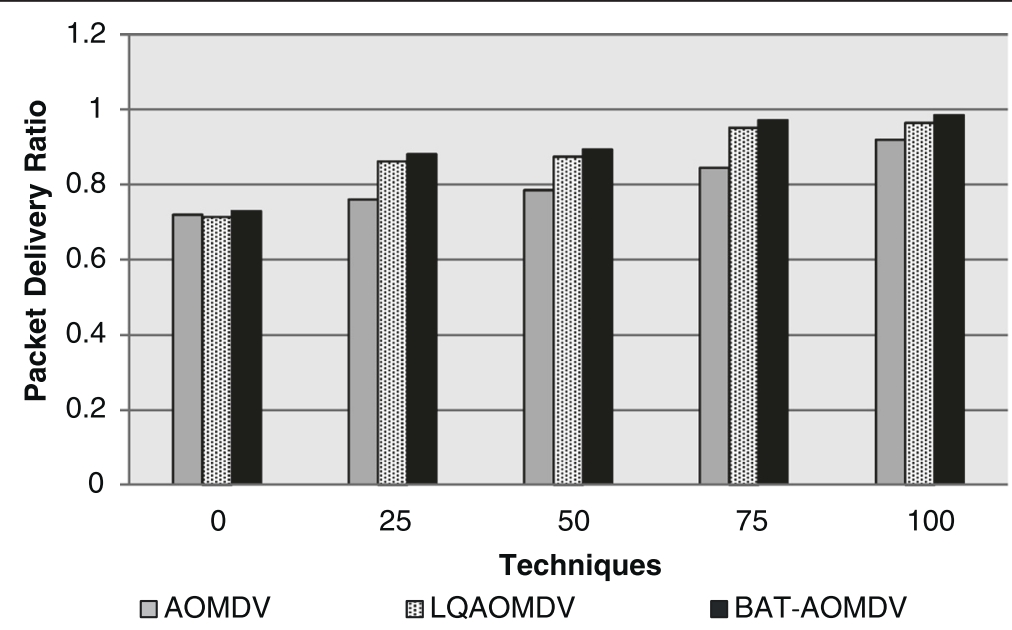

Fig. 2 Packet delivery ratio (PDR) 
Table 3 Delays obtained in the network

\begin{tabular}{llll}
\hline \multicolumn{4}{c}{$\begin{array}{l}\text { Average end-to-end } \\
\text { delay in seconds }\end{array}$} \\
$\begin{array}{llll}\text { Node pause time } \\
\text { in seconds }\end{array}$ & AOMDV & LQ-AOMDV & BAT-AOMDV \\
0 & 0.8712 & 0.9245 & 0.8268 \\
25 & 0.5107 & 0.4711 & 0.485 \\
50 & 0.4035 & 0.3318 & 0.377 \\
75 & 0.2815 & 0.3076 & 0.2606 \\
100 & 0.207 & 0.265 & 0.1928 \\
& & & 0 \\
& Average jitter in & & \\
Node pause time & AOMDV & LQ-AOMDV & BAT-AOMDV \\
in seconds & & & \\
0 & 0.003933 & 0.004091 & 0.004365 \\
25 & 0.00218 & 0.001128 & 0.002497 \\
50 & 0.002012 & 0.001624 & 0.001259 \\
75 & 0.000812 & 0.001221 & 0.00057 \\
100 & 0.001087 & 0.000785 & 0.000754 \\
\hline
\end{tabular}

\section{$\mathrm{S} \rightarrow \mathrm{N} 3 \rightarrow \mathrm{N} 4 \rightarrow \mathrm{N} 5 \rightarrow \mathrm{D}$}

Based on network parameters, the best solution obtained is the route $\mathrm{S} \rightarrow \mathrm{N} 1 \rightarrow \mathrm{N} 4 \rightarrow \mathrm{D}$, then a local search is initiated such that part of the route tree is changed. For example, a feasible solution obtained from local search can be represented by

$$
\mathrm{S} \rightarrow \mathrm{N} 1 \rightarrow \mathrm{N} 6 \rightarrow \mathrm{D}
$$

Since the solution space is very large, new solutions are also created randomly every iteration. The BAT parameters used in this work is given in Table 1.

\section{Results and discussion}

We assume that mobile nodes are distributed uniformly with node density $d$ inside a rectangular area of size $\mathrm{U} \times$ $\mathrm{V}$. We also assume that there are $N$ nodes in the network. In the free space, propagation model used the signal attenuates as $\frac{1}{r^{2}}$, where $r$ is the distance between the nodes. For the MAC layer protocol, IEEE 802.11 with the distributed coordination function (DCF) is used. The rectangular region is chosen to increase the average route length in the simulation. All nodes move at the same average speed of 4-8 $\mathrm{m} / \mathrm{s}$ with a transmission distance of $250 \mathrm{~m}$. The node's pause time is increased in our simulation and the impact studied. Zero pause time represents the node is in continuous motion. For traffic, constant bit rate (CBR) is used with 10 source and 10 destination nodes selected randomly with an inter packet arrival time of $200 \mathrm{~ms}$ with the size of each packet being 512 bytes. The buffer size of each node is set to 65,536 bytes. Simulations were carried out using OPNET simulator and the proposed technique compared with AOMDV and Link Quality (LQ)-AOMDV, the non optimized version of link quality-based AOMDV. The simulation parameters used in this work is shown in Table 2.

Figure 2 shows the obtained packet delivery ratio.

From Fig. 2, it is observed that the packet delivery ratio (PDR) of proposed BAT-AOMDV improves by $10.2 \%$ compared to AOMDV and by $2.17 \%$ compared to LQ-AOMDV under different node pause time. Zero node pause time represents that the nodes are continuously mobile. Good link quality of the obtained route reduced the packet drop substantially with increasing node pause time. Table 3 shows the end-to-end delay and jitter obtained by simulations.

From the table, it can be observed that BAT-AOMDV overcomes the limitation of LQ-AOMDV by reducing

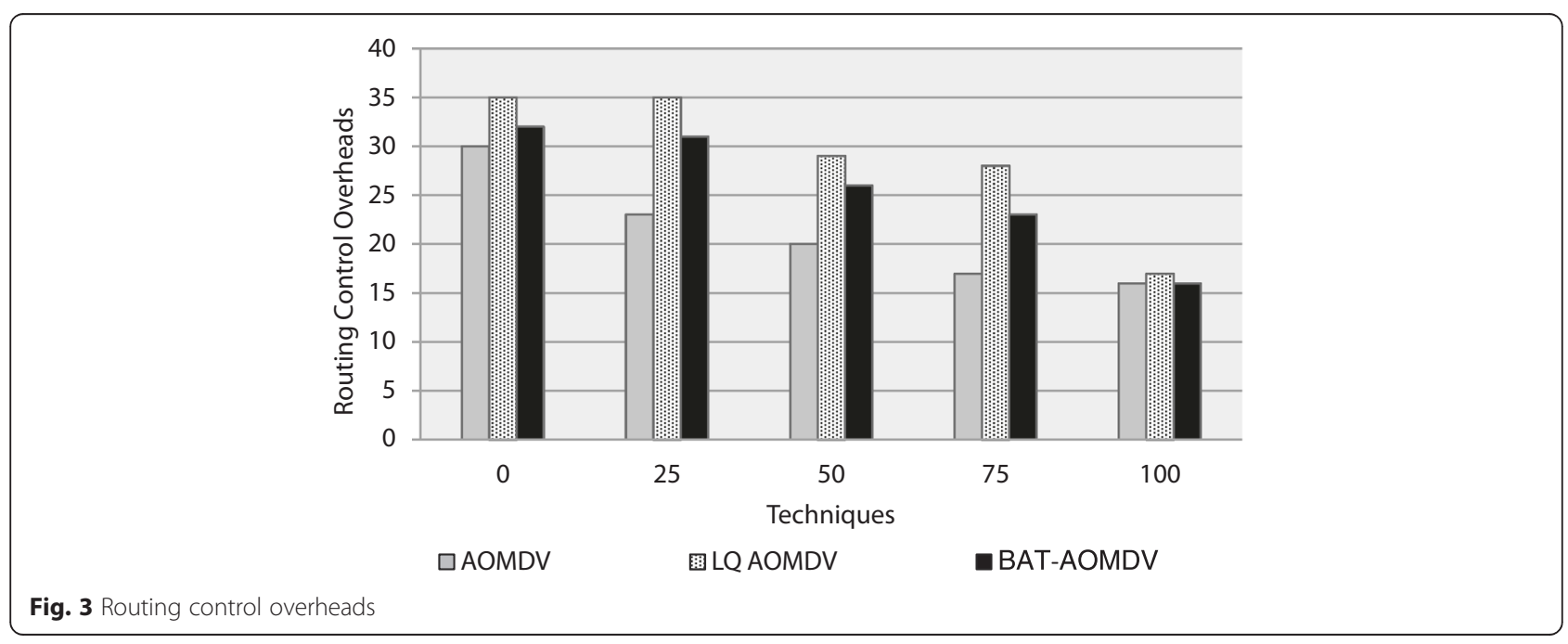




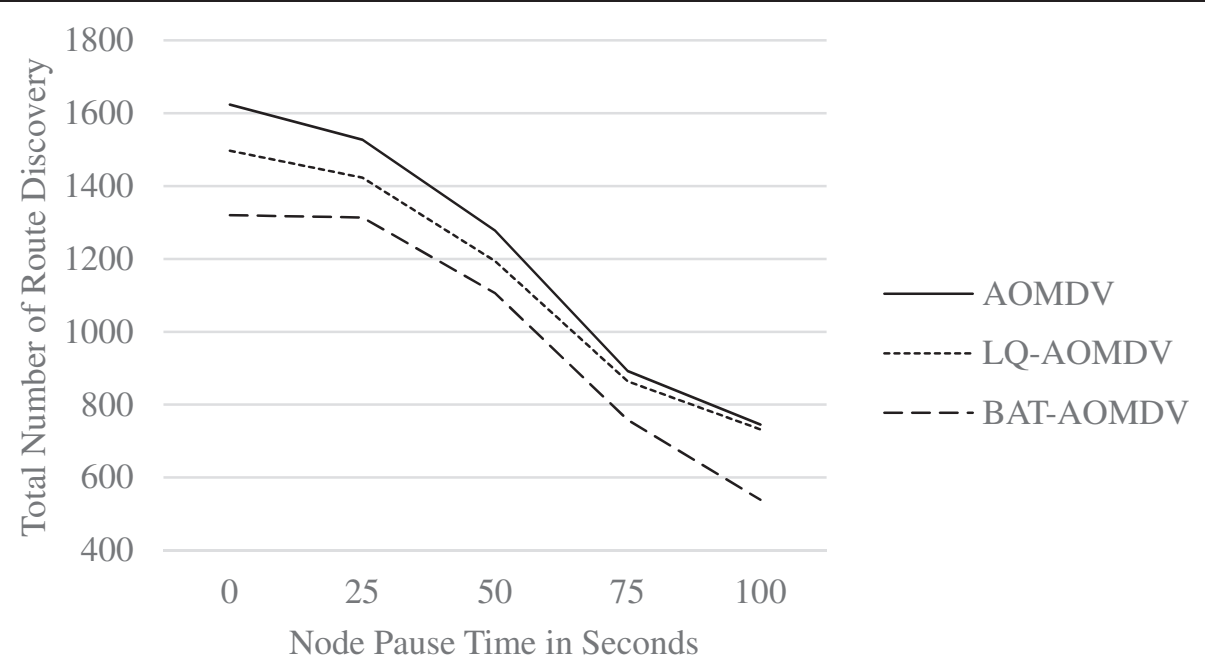

Fig. 4 Total number of route discovery

the average end-to-end delay by an average of $7.1 \%$ and compared to AOMDV by $5.96 \%$. Similar improvements in jitter can also be observed. This also shows that route discovery is suboptimal. Figure 3 shows the routing control overheads for different node pause time.

Though AOMDV scores well in lowering the control packet overhead, there is no significant increase in control packet overheads for BAT-AOMDV at higher node pause times. However, the objective of decreasing control packet overhead by route optimization is obvious. Figure 4 shows the total number of route discovery.

From Fig. 4, it is observed that BAT-AOMDV is able to discover more routes for its objective function by random selection. The convergence characteristic of proposed algorithm is shown in Fig. 5.

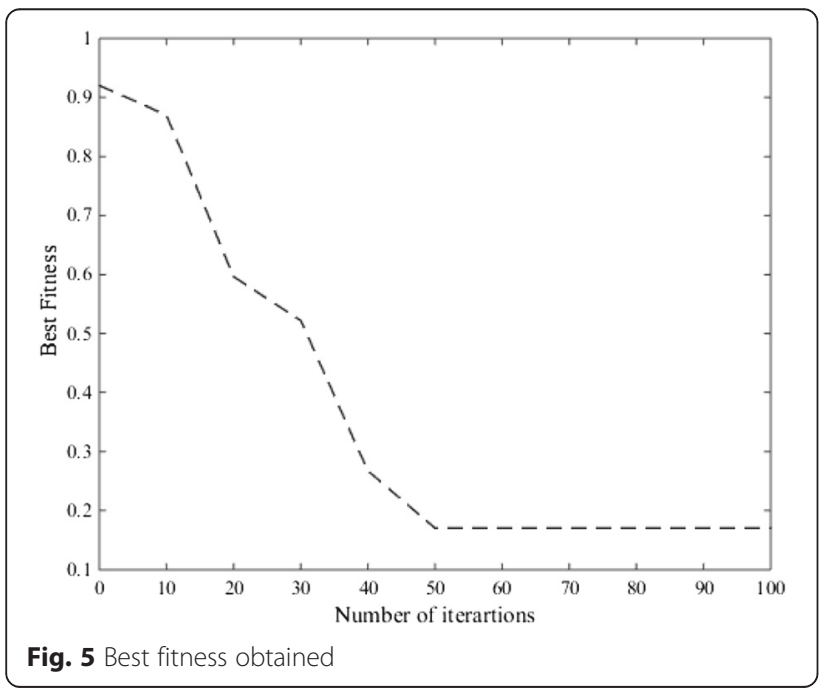

Bat meta-heuristic is able to converge faster and was able to find an optimal solution in less than 50 iterations.

\section{Conclusions}

This work proposed improvements in AOMDV based on Link Quality. A link estimation algorithm-based AOMDV was proposed (LQ-AOMDV). The proposed LQ-AOMDV was optimized to obtain optimal solutions. A novel objective function was proposed to balance the load across the network and improve the network performance. Simulations results show that PDR of proposed BAT-AOMDV improved when compared to AOMDV and LQ-AOMDV. Average BAT-AOMDV values increased by $10.2 \%$ when compared to AOMDV and increased by $2.17 \%$ when compared to LQ-AOMDV. Further investigation is necessary to lower end-to-end delay.

\section{Competing interests}

Both authors declare that they have no competing interests.

\section{Author details}

${ }^{1}$ Department of Electronics and Communication Engineering, Indira Gandhi College of Engineering and Technology for Women, Tamil Nadu, India. ${ }^{2}$ Department of Computer Science and Engineering, Thangavelu Engineering College, Tamil Nadu, India.

Received: 27 December 2014 Accepted: 13 May 2015

Published online: 18 June 2015

\section{References}

1. KS Chung, JE Lee, Design and Development of m-Learning Service Based on 3G Cellular Phones. JIPS 8(3), 521 (2012)

2. H Luo, ML Shyu, Quality of service provision in mobile multimedia-a survey. Human-centric Computing and Information Sciences 1(1), 1-15 (2011)

3. JKY Ng, Ubiquitous healthcare: healthcare systems and applications enabled by mobile and wireless technologies. Journal of Convergence 3(2), 31-36 (2012)

4. Gawande, A. (2013). Performance analysis of DSR protocol under sinkhole attack in MANETs. International Journal, 1(4). 
5. R Sumathi, MG Srinivas, A survey of QoS based routing protocols for wireless sensor networks. J Inf Process Syst 8(4), 589-602 (2012)

6. AU Bandaranayake, V Pandit, DP Agrawal, Indoor link quality comparison of IEEE 802.11 a channels in a multi-radio mesh network testbed. J Inf Process Syst 8(1), 1-20 (2012)

7. Li, X., Mitton, N., Nayak, A., \& Stojmenovic, I. (2012). Achieving load awareness in position-based wireless ad hoc routing. Journal of Convergence, 3(3).

8. Chien, F. T., Wu, K. G., Chan, Y. W., Chang, M. K., \& Su, Y. S. (2014). An Effective Routing Protocol with Guaranteed Route Preference for Mobile Ad Hoc Networks. International Journal of Distributed Sensor Networks, 2014. Hindawi Publishing Corporation International Journal of Distributed Sensor Networks Volume 2014, Article ID 532049, 18 pages http://dx.doi.org/ $10.1155 / 2014 / 532049$

9. CE Perkins, Ad hoc Networking, 2008. Addison-Wesley Professional. Addison-Wesley Professional @2008 ISBN:0321579070 9780321579072

10. CE Perkins, EM Royer, Ad-hoc on-demand distance vector routing. Paper presented at the Second IEEE Workshop on Mobile Computing Systems and Applications, 1999. Proceedings. WMCSA'99, IEEE, 25-26 February 1999, pp. 90-100.

11. Taneja, S., Kush, A., \& Makkar, A. (2010). Experimental Analysis of DSR, AODV using Speed and Pause time. International Journal of Innovation, Management and Technology, 1(5), 2010-0248. 453-458 (2010)

12. GUO Peng-cheng, Research of AODV Routing Protocol for Ad Hoc Networks. Science Technology and Engineering 18, 023 (2013)

13. L Abusalah, A Khokhar, M Guizani, A survey of secure mobile ad hoc routing protocols. Commun Surveys Tuts 10(4), 78-93 (2008)

14. MK Marina, SR Das, On-demand multipath distance vector routing in ad hoc networks, Paper presented at the Ninth International Conference on Network Protocols, 2001, IEEE, 11-14 November 2001, pp. 14-23

15. RK Kapoor, MA Rizvi, S Sharma, MM Malik, Exploring Multi Path routing Protocols in Mobile Ad hoc Networks. Journal of Computer and Mathematical Sciences 2(5), 693-779 (2011)

16. SV Mallapur, SR Patil, Stable backbone based multipath routing protocol for mobile ad-hoc networks. Paper presented at the 2013 International Conference on Circuits, Power and Computing Technologies (ICCPCT), IEEE, Nagercoil, 20-21 March 2013 pp. 1105-1110.

17. $M Y u, W$ Liu, T Xing, Link availability modeling for routing algorithms to reduce the link break time in MANETs. Paper presented at the 2011 IEEE International Conference on Networking, Sensing and Control (ICNSC), IEEE, Delft, 11-13 April 2011, pp. 115-120.

18. M Yu, J Bao, A ranging-based link availability routing algorithm for MANETs. Paper presented at the 2010 IEEE on Wireless Communications and Networking Conference (WCNC), IEEE, Sydney, Australia, 18-21 April 2010, pp. 1-6.

19. T Yelemou, New approach to improve the robustness of AOMDV protocol. Paper presente at the 2013 10th International Multi-Conference on Systems, Signals and Devices (SSD), IEEE, Hammamet, 18-21 March 2013, pp. 1-6.

20. M Obaidat, MA Ali, MS Obaidat, S Obeidat, I Shahwan, A novel multipath routing protocol for MANETs. Paper presented at the 2011 7th International Conference on Wireless Communications, Networking and Mobile Computing (WiCOM), IEEE, Wuhan, 23-25 September 2011, pp. 1-6.

21. X Chen, HM Jones, D Jayalath, Channel-aware routing in MANETs with route handoff. IEEE Trans Mobile Comput 10(1), 108-121 (2011)

22. Mazumdar, A. P., Surange, A., \& Sairam, A. S. (2014, January). Providing Stable Routes in Mobile Ad Hoc Networks. In Proceedings of Ninth International Conference on Wireless Communication and Sensor Networks (pp. 51-60). Springer India.

23. J Cai, K Liu, An improved AOMDV routing protocol based on prediction of link stability. Paper presented at the Fourth International Conference on Machine Vision (ICMV 11), International Society for Optics and Photonics, Singapore, Singapore, 9 December 2011, p. 83500V.

24. R Singh, S Gupta, EE-AODV: Energy Efficient AODV routing protocol by Optimizing route selection process. IJRCCT 3(1), 158-163 (2014)

25. Khimsara, S., Kambhatla, K. K., Hwang, J., Kumar, S., \& Matyjas, J. D. (2010). AM-AOMDV: adaptive multi-metric ad-hoc on-demand multipath distance vector routing. In Ad Hoc Networks (pp. 884-895). Springer Berlin Heidelberg.

26. M Gunes, U Sorges, I Bouazizi, ARA-the ant-colony based routing algorithm for MANETs. Paper presented at the International conference on Parallel Processing Workshops, 2002. Proceedings, IEEE, 2002, pp. 79-85.

27. L Liu, G Feng, Swarm intelligence based node-disjoint multi-path routing protocol for mobile ad hoc networks. Paper presented at the 2005 Fifth
International Conference on Information, Communications and Signal Processing, IEEE, Bangkok, 2005, pp. 598-602.

28. Ziane, S., \& Melouk, A. (2005, October). A swarm intelligent multi-path routing for multimedia traffic over mobile ad hoc networks. In Proceedings of the 1st ACM international workshop on Quality of service \& security in wireless and mobile networks (pp. 55-62). ACM.

29. Xiang, L., Luo, J., and Vasilakos, A. (2011, June). Compressed data aggregation for energy efficient wireless sensor networks. Paper presented at the 2011 8th annual IEEE communications society conference on Sensor, mesh and ad hoc communications and networks (SECON), IEEE, Salt Lake City, UT, 27-30 June 2011, pp. 46-54.

30. Vasilakos, A., Ricudis, C., Anagnostakis, K., Pedryca, W., and Pitsillides, A. (1998, May). Evolutionary-fuzzy prediction for strategic QoS routing in broadband networks. In Fuzzy Systems Proceedings, 1998. IEEE World Congress on Computational Intelligence., The 1998 IEEE International Conference on (Vol. 2, pp. 1488-1493). IEEE

31. Pitsillides, A., Stylianou, G., Pattichis, C. S., Sekercioglu, A., and Vasilakos, A. (2000, March). Bandwidth allocation for virtual paths (BAVP): investigation of performance of classical constrained and genetic algorithm based optimisation techniques. In INFOCOM 2000. Nineteenth Annual Joint Conference of the IEEE Computer and Communications Societies. Proceedings. IEEE (Vol. 3, pp. 1501-1510). IEEE

32. YS Yen, HC Chao, RS Chang, A Vasilakos, Flooding-limited and multi-constrained QoS multicast routing based on the genetic algorithm for MANETs. Math Comput Model 53(11), 2238-2250 (2011)

33. Y Song, L Liu, H Ma, AV Vasilakos, A biology-based algorithm to minimal exposure problem of wireless sensor networks. IEEE trans. Netw. Serv. Manage. 11(3), 417-430 (2014)

34. L Liu, Y Song, H Zhang, H Ma, AV Vasilakos, Physarum optimization: A biology-inspired algorithm for the steiner tree problem in networks. IEEE Trans. Comput. 64(3), 819-832 (2015)

35. Li, P., Guo, S., Yu, S., \& Vasilakos, A. V. (2012, March). CodePipe: An opportunistic feeding and routing protocol for reliable multicast with pipelined network coding. In INFOCOM, 2012 Proceedings IEEE (pp. 100-108). IEEE.

36. Y Zeng, K Xiang, D Li, AV Vasilakos, Directional routing and scheduling for green vehicular delay tolerant networks. Wireless Network 19(2), 161-173 (2013)

37. A Cianfrani, V Eramo, M Listanti, M Polverini, AV Vasilakos, An OSPF-integrated routing strategy for QoS-aware energy saving in IP backbone networks. IEEE Trans. Netw. Serv. Manage 9(3), 254-267 (2012)

38. C Busch, R Kannan, AV Vasilakos, Approximating Congestion+ Dilation in Networks via "Quality of Routing" Games. IEEE. Trans. Comput. 61(9), 1270-1283 (2012)

39. T Spyropoulos, RN Rais, T Turletti, K Obraczka, A Vasilakos, Routing for disruption tolerant networks: taxonomy and design. Wireless Network 16(8), 2349-2370 (2010)

40. M Youssef, M Ibrahim, M Abdelatif, L Chen, AV Vasilakos, Routing metrics of cognitive radio networks: A survey. IEEE Commun. Surv. Tut. 16(1), 92-109 (2014)

41. H Cheng, N Xiong, AV Vasilakos, LT Yang, G Chen, X Zhuang, Nodes organization for channel assignment with topology preservation in multi-radio wireless mesh networks. Ad Hoc Networks 10(5), 760-773 (2012)

42. Y Yao, Q Cao, AV Vasilakos, EDAL: an energy-efficient, delay-aware, and lifetime-balancing data collection protocol for wireless sensor networks. Paper presented at the 2013 IEEE 10th international conference on Mobile ad-hoc and sensor systems (MASS), IEEE, Hangzhou, 14-16 October 2013, pp. 182-190.

43. X Wang, AV Vasilakos, M Chen, Y Liu, TT Kwon, A survey of green mobile networks: Opportunities and challenges. Mobile Network Appl. 17(1), 4-20 (2012)

44. K Han, J Luo, Y Liu, AV Vasilakos, Algorithm design for data communications in duty-cycled wireless sensor networks: A survey. IEEE Commun Mag 51(7), 107-113 (2013)

45. N Xiong, AV Vasilakos, LT Yang, L Song, Y Pan, R Kannan, Y Li, Comparative analysis of quality of service and memory usage for adaptive failure detectors in healthcare systems. IEEE J Sel Area Comm 27(4), 495-509 (2009)

46. P Li, S Guo, S Yu, AV Vasilakos, Reliable multicast with pipelined network coding using opportunistic feeding and routing. IEEE T Parall Distr 25(12), 3264-3273 (2014)

47. XM Zhang, Y Zhang, F Yan, AV Vasilakos, Interference-based Topology Control Algorithm for Delay-constrained Mobile Ad hoc Networks. IEEE Trans. Mobile Comput. 14(4), 742-754 (2015) 
48. A Vasilakos et al., Evolutionary-fuzzy prediction for strategic QoS routing in broadband networks. Paper presented at the 1998 IEEE International Conference (Volume 2) on Fuzzy Systems Proceedings, IEEE, Anchorage, AK, 4-9 May 1998, pp. 1488-1493

49. Znati, T. (1999). A path availability model for wireless ad-hoc networks. In Wireless communications and networking conference, 1999. WCNC. 1999 IEEE (pp. 35-40). IEEE.

50. TF Znati, A mobility-based framework for adaptive clustering in wireless ad hoc networks. IEEE J Sel Area Comm 17(8), 1466-1487 (1999)

51. He, D. J., Jiang, S. M., \& Rao, J. Q. (1999, November). Prediction of link availability in wireless ad-hoc networks. In The 1999 Singapore Pervasive Computing Conference (pp. 17-19).

52. He, D., Jiang, S., \& Rao, J. (2000, April). A Link Availability Prediction Model for Wireless Ad Hoc Networks. In ICDCS Workshop on Wireless Networks and Mobile Computing (pp. D7-D11).

53. Su, W., \& Gerla, M. (1999). IPv6 flow handoff in ad hoc wireless networks using mobility prediction. In Global Telecommunications Conference, 1999. GLOBECOM'99 (Vol. 1, pp. 271-275). IEEE.

54. Jiang, S., He, D., \& Rao, J. (2001). A prediction-based link availability estimation for mobile ad hoc networks. In INFOCOM 2001. Twentieth Annual Joint Conference of the IEEE Computer and Communications Societies. Proceedings. IEEE (Vol. 3, pp. 1745-1752). IEEE

55. MF Maghrebi, MR Aghaebrahimi, H Taherian, M Attari, Determining the amount and location of leakage in water supply networks using a neural network improved by the Bat optimization algorithm. J Civil Eng Urban 4(3), 322-327 (2014)

\section{Submit your manuscript to a SpringerOpen ${ }^{\circ}$} journal and benefit from:

- Convenient online submission

- Rigorous peer review

- Immediate publication on acceptance

- Open access: articles freely available online

- High visibility within the field

- Retaining the copyright to your article 